\title{
Computational fluid dynamics as a risk assessment tool for aneurysm rupture
}

\author{
Yuichi Murayama, MD, ${ }^{1,2}$ Soichiro Fujimura, MS, ${ }^{2,3}$ Tomoaki Suzuki, MD, PhD, ${ }^{4}$ and \\ Hiroyuki Takao, MD, PhD ${ }^{1-3}$
}

\begin{abstract}
Departments of ${ }^{1}$ Neurosurgery and ${ }^{2}$ Innovation for Medical Information Technology, The Jikei University School of Medicine, Tokyo; ${ }^{3}$ Graduate School of Mechanical Engineering, Tokyo University of Science, Tokyo; and ${ }^{4}$ Department of Neurosurgery, Brain Research Institute, Niigata University, Niigata, Japan
\end{abstract}

OBJECTIVE The authors reviewed the clinical role of computational fluid dynamics (CFD) in assessing the risk of intracranial aneurysm rupture.

METHODS A literature review was performed to identify reports on CFD assessment of aneurysms using PubMed. The usefulness of various hemodynamic parameters, such as wall shear stress (WSS) and the Oscillatory Shear Index (OSI), and their role in aneurysm rupture risk analysis, were analyzed.

RESULTS The authors identified a total of 258 published articles evaluating rupture risk, growth, and endovascular device assessment. Of these 258 articles, 113 matching for CFD and hemodynamic parameters that contribute to the risk of rupture (such as WSS and OSI) were identified. However, due to a lack of standardized methodology, controversy remains on each parameter's role.

CONCLUSIONS Although controversy continues to exist on which risk factors contribute to predict aneurysm rupture, CFD can provide additional parameters to assess this rupture risk. This technology can contribute to clinical decisionmaking or evaluation of efficacy for endovascular methods and devices.

https://thejns.org/doi/abs/10.3171/2019.4.FOCUS19189

KEYWORDS intracranial aneurysm; rupture; computational fluid dynamics; hemodynamics

$\mathrm{P}$ REDICTION of aneurysm rupture is complex and difficult. In addition to clinical and morphological information, computational fluid dynamics (CFD) parameters have been reported to be valuable predictors for rupture. Numerous hemodynamic parameters, such as wall shear stress (WSS) and the Oscillatory Shear Index (OSI), have been introduced and studied during the last decade. These hemodynamic parameters have been identified as contributing to the risk of rupture. $13,4,4,6,13,17,24,27-31$, 37,39-41 However, due to a lack of standardized methodology, controversy remains on each parameter's role. In this review article, we analyze the current status of CFD research that may play an important role in clinical neurosurgery, and specifically evaluate the current role of CFD as a predictor of cerebral aneurysm rupture.

\section{Methods}

A PubMed search identified a total of 258 published articles evaluating aneurysm rupture risk, growth, and endovascular device assessment, and 113 of these articles matched for CFD and aneurysm rupture.

\section{Results and Discussion \\ CFD Method: How to Analyze Aneurysm Data}

Details of the common process of CFD modeling are provided in Fig. 1. To conduct precise CFD analysis, a high-quality 3D data set is fundamental and high-resolution images such as digital subtraction angiography or CT angiography are widely used. After obtaining the DICOM data set, the computational volumetric mesh is generated

ABBREVIATIONS CFD = computational fluid dynamics; FD = flow diverter; FSI = fluid structure interaction; ICA = internal carotid artery; $\mathrm{MCA}=$ middle cerebral artery; OSI = Oscillatory Shear Index; PD = pressure difference; PLc = pressure loss coefficient; $S T L$ = stereolithography; WSS = wall shear stress.

SUBMITTED February 28, 2019. ACCEPTED April 23, 2019.

INCLUDE WHEN CITING DOI: 10.3171/2019.4.FOCUS19189. 


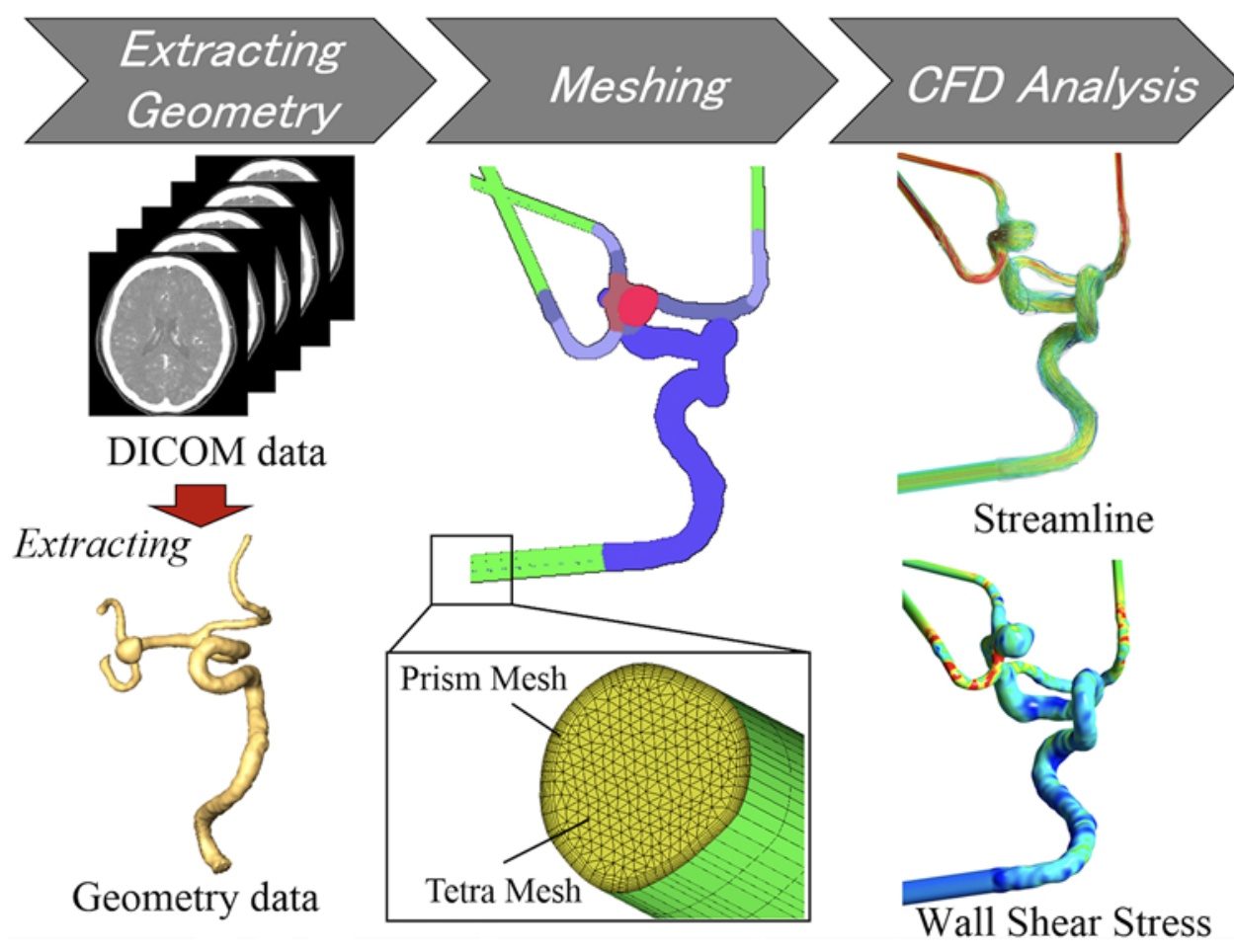

FIG. 1. Workflow of computational analysis.

based on the generated artery and aneurysm geometry data. When CFD simulations for prediction of aneurysm rupture are performed, it is important to know whether or not the geometry was reconstructed from prerupture aneurysm imaging, because the geometry might have changed after rupture, and that will produce hemodynamic differences.

\section{Flow Condition}

Due to the difficulty of obtaining patient-specific data, physiological information such as blood pressure, flow velocity, blood viscosity, and heart rate are usually fixed as a uniform condition for the CFD analysis. Although in some studies fluid structure interaction (FSI) analysis was applied to simulate vessel transformation during pulsation, and thus the elasticity of the blood vessel was considered, in the majority of the studies the vascular wall was usually considered to be rigid. FSI analysis contains very complex computational techniques and their results also depend on the thickness or elastic modulus of the vessel, which is difficult to obtain by common diagnostic imaging devices.

Most studies including a large number of aneurysms considered blood as a Newtonian fluid with specific values of density and viscosity. Very few studies accounted for the effect of blood viscosity. Many researchers used standardized blood viscosity.

In flow analysis, the flow field was mostly assumed to be incompressible laminar flow because the Reynolds number (based on vessel diameter and flow speed) was in the range of several hundreds. After applying computational preconditions, including assumed blood material properties and boundary conditions, the blood flow was simulated, solving the so-called "Navier-Stokes equations."

\section{Analysis of Hemodynamic Parameters}

As a next step, some specific hemodynamic parameters, such as WSS or OSI, were calculated. Table 1 shows recent key articles and the parameters they have analyzed. Finally, visualization software is used for a better understanding of blood flow by illustrating streamline and hemodynamic parameters, such as pressure and WSS.

\section{Wall Shear Stress}

WSS is a frictional force on the arterial wall produced by blood flow in a direction toward a local tangent plane, as described in the following equation:

$$
W S S=\mu\left(\frac{\partial u}{\partial y}\right)_{y=0}
$$

in which $\mu$ is the dynamic viscosity, $u$ is the blood velocity parallel to the wall, and $y$ is the distance from the arterial wall. WSS is one of the most popular hemodynamic parameters in CFD research and has been used to investigate the behavior of aneurysms including rupture, growth, and initiation. There is no doubt that this small frictional force contributes to biological reactions occurring on the parent artery or aneurysmal wall. However, controversial results of both high and low WSS have been reported to be correlated with aneurysm rupture.

\section{Oscillatory Shear Index}

The OSI measures the directional changes of WSS dur- 
TABLE 1. Recent key articles and parameters with CFD for aneurysm rupture

\begin{tabular}{|c|c|c|c|c|c|c|c|}
\hline \multirow[b]{2}{*}{ Authors \& Year } & \multicolumn{2}{|r|}{ CFD Parameters for Aneurysm Rupture } & \multirow[b]{2}{*}{ Location } & \multirow[b]{2}{*}{ Sensitivity } & \multirow[b]{2}{*}{ Specificity } & \multirow[b]{2}{*}{ AUC } & \multirow{2}{*}{$\begin{array}{c}\text { Analyzed } \\
\text { Cases }\end{array}$} \\
\hline & WSS & Other Possible Parameters & & & & & \\
\hline Castro et al., 2009 & High & Small impaction zone & $\mathrm{ACoA}$ & - & - & - & 26 \\
\hline Xiang et al., 2011 & Low & OSI & Many & 0.75 & 0.84 & 0.89 & 119 \\
\hline Cebral et al., $2011^{6}$ & - & $\begin{array}{l}\text { Concentrated inflow, small impingement, } \\
\text { complex flow, unstable flow }\end{array}$ & Many & - & - & - & 210 \\
\hline Qian et al., 2011 & - & EL & ICPcom & - & - & - & 30 \\
\hline Takao et al., 2012 & Low & PLC & ICPcom/MCA & $0.833 / 0.714$ & $0.818 / 0.721$ & $0.845 / 0.792$ & 100 \\
\hline Miura et al., 2013 & Low & - & MCA & 0.698 & 0.698 & 0.723 & 106 \\
\hline Jing et al., 2015 & Low & LSA, OSI & Many & - & - & 0.924 & 155 \\
\hline Schneiders et al., 2015 & - & High risk location, daughter sacs & Many & - & - & 0.72 & 117 \\
\hline Zhang et al., 2016 & Low & Age, irregular shape, LSA & ICPcom & - & - & - & 173 \\
\hline Xiang et al., 2016 & Low & OSI, UI, NSI, EL, LSA, RRT, NV & Many & - & - & 0.831 & 204 \\
\hline
\end{tabular}

$\mathrm{ACOA}=$ anterior communicating artery; $\mathrm{AUC}=$ area under the curve; $\mathrm{EL}=$ energy loss; ICPcom = internal carotid posterior communicating artery; $\mathrm{LSA}=$ low wall shear area percentage; $\mathrm{NSI}$ = non-sphericity index; $\mathrm{NV}$ = number of vortices; $\mathrm{RRT}$ = relative residence time; $\mathrm{UI}$ = undulation index.

ing the cardiac cycle. The OSI becomes larger with larger angle changes of WSS direction and is often used to describe the disturbance of a flow field. Previous studies reported that a higher OSI was observed in ruptured than in unruptured aneurysms, or high OSI corresponded to the rupture point. OSI is described in the following equation:

$$
O S I=\frac{1}{2}\left(1-\frac{\left|\int_{0}^{T} \boldsymbol{w s s}_{i} d t\right|}{\int_{0}^{T}\left|\boldsymbol{w s} \boldsymbol{s}_{i}\right| d t}\right)
$$

in which wss $_{i}$ is the instantaneous WSS vector, $t$ is time, and $T$ is the duration of the cycle.

\section{Pressure Difference}

The pressure difference (PD) was defined as the degree of pressure elevation at the aneurysm wall and calculated by subtracting $P_{\text {ave }}$ from $P$. This value was normalized by dividing it by the dynamic pressure at the aneurysm inlet side. PD is defined as shown in the following formula:

$$
P D=\frac{P-P_{\text {ave }}}{\frac{1}{2} \rho v_{\text {in }}^{2}}
$$

where $P$ is local pressure, $P_{\text {ave }}$ is average pressure in the aneurysm domain, $\rho$ is density of blood, and $v_{i n}$ is the mean velocity of the aneurysm inlet.

\section{Pressure Loss Coefficient}

The pressure loss coefficient (PLc) calculates pressure loss associated with the shape of the aneurysms and obstacles of blood flow. This parameter indicates whether the blood flows "easily" (without resistance) through the aneurysms, or what is the quantitative expression of this "easiness" (i.e., PLc decreases if the shape of the vessels and aneurysms facilitates the easy flow of blood). PLc is obtained by the following equation:

$$
P L C=\frac{\left(\frac{1}{2} \rho v_{\text {in }}^{2}+P_{\text {in }}\right)-\left(\frac{1}{2} \rho v_{\text {out }}^{2}+P_{\text {out }}\right)}{\frac{1}{2} \rho v_{\text {in }}^{2}}
$$

where $\rho$ is the density of blood, $P$ is static pressure, and $v$ is the flow velocity. The suffixes in and out indicate the inlet and outlet of the control volume, which is defined at the cross-section at a distance $1 \mathrm{~mm}$ from the aneurysmal neck or bifurcation point. Unlike the parameters that directly evaluate the force acting on the walls of aneurysms such as WSS, OSI, or PD, this parameter describes the hydrodynamic pressure loss due to blood passing through the parent artery and the aneurysmal sac.

\section{Hemodynamic Parameters and Aneurysm Status}

Some researchers reported that a higher WSS was observed in ruptured compared to unruptured aneurysms, but others reported the opposite finding. Cebral et al. ${ }^{5}$ reported that high WSS was related to aneurysmal rupture. Hemodynamics interacting with the thickness and hardness of the walls was evaluated at the identified rupture points of 9 ruptured aneurysms. ${ }^{7}$ The study concluded that high WSS at thin and hard aneurysmal wall areas was most involved with rupture. Other researchers have reported that aneurysms ruptured because blood flow impingement on the aneurysmal wall produced high WSS. ${ }^{14}$ Conversely, some reports indicated that aneurysms ruptured because they could no longer sustain the arterial pressure due to the degeneration and thinning of the arterial walls caused by low WSS. ${ }^{19}$ Omodaka et al. ${ }^{25}$ performed CFD simulations of 6 middle cerebral artery (MCA) aneurysms, with their rupture points identified during craniotomy. They reported that the time-averaged WSS was statistically significantly lower at rupture points than at nonruptured aneurysmal walls. Fukazawa et al. ${ }^{12}$ also reported that the WSS was statistically significantly lower at the rupture points of aneurysms compared to the parent artery areas, observed in CFD simulations of 12 cases of ruptured aneurysms. Additionally, 
in recent years, the results of large-scale CFD studies on more than 100 ruptured and unruptured aneurysms have also been reported. Zhang et al. ${ }^{41}$ and Xiang et al ${ }^{40}$ performed CFD analyses and statistical tests for a total of 173 and 204 aneurysms, respectively, including ruptured and unruptured aneurysms. They also reported that aneurysmal rupture had a strong relation to hemodynamic and morphological parameters, such as low WSS, high OSI, and size ratio.

Because of these controversial reports on WSS (i.e., both low and high WSS reported as risk factors for rupture), Meng et al. ${ }^{23}$ published a review article unifying the results from previous reports with their own. They indicated that high WSS and a positive WSS gradient can trigger mural cell-mediated destructive remodeling, and low WSS and a high OSI can trigger inflammatory cell-mediated destructive remodeling. First, high WSS at the blood flow jet impingement zone is related to the initiation of aneurysms. After aneurysm initiation, its growth process differs depending on whether WSS is high or low. If the WSS is high, it causes degeneration of the cellular matrix and cell apoptosis, and aneurysms may rupture even if they were small in size. However, if the WSS is low, the aneurysm may become larger because inflammation caused by slow recirculation and a disturbed flow environment promotes the formation of atherosclerotic plaques, which exacerbate the effects on inflammatory cells. Finally, aneurysms will rupture or stabilize depending on the temporal and spatial WSS alterations accompanied by geometrical changes of the aneurysms. Meng et al. indicated that the cause of the leading controversial reports on WSS depended on the difference in the natural history of each analyzed aneurysm. More detailed assessment of WSS might be of benefit to understand the result of CFD analysis for ruptured aneurysms.

However, in most studies of ruptured and unruptured aneurysms, CFD has been performed on geometries reconstructed from the arterial images taken after their rupture. $3,4,6,17,20,24,27,28,30,37,39-41$ Such studies were based on the assumption that ruptured and unruptured aneurysms have different value in CFD parameters. To predict future rupture, the use of prerupture aneurysm imaging is mandatory, and some researchers evaluated CFD values using prerupture imaging. Hodis et al ${ }^{16}$ reported a rare periprocedural rupture case during cerebral angiography of an unruptured aneurysm. They found high WSS and an impinging jet at the rupture point. Kono and Terada ${ }^{21}$ reported on mirror-image aneurysms in which one ruptured during the observation. The results of comparing the CFD analysis on images that were taken before and after rupture showed that the aneurysm shape change due to rupture caused a $20 \%-30 \%$ change of WSS. Thus, they concluded that CFD studies comparing unruptured aneurysms with those after rupture may not yield valid estimations on their rupture risk.

Qian et al..$^{27}$ reported CFD analysis of prerupture imaging in which rupture occurred during follow-up of observed unruptured aneurysms. They found high energy loss was a risk factor for rupture. Takao et al. ${ }^{37}$ analyzed the largest number of ruptured and unruptured aneurysms using the images taken before rupture for ruptured
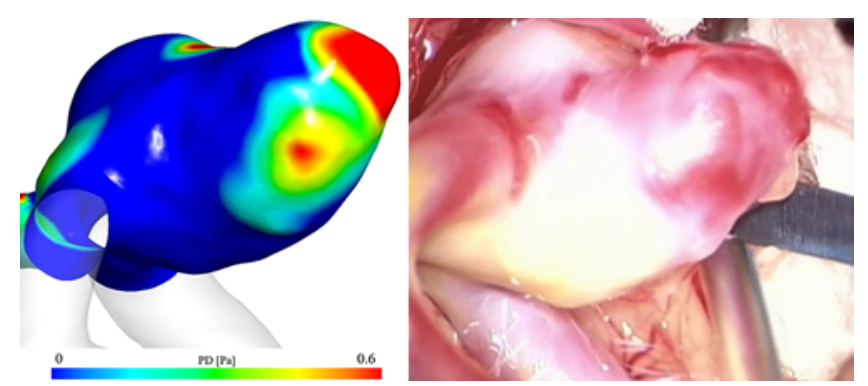

FIG. 2. Images obtained in a 75-year-old woman who presented with a growing right MCA unruptured aneurysm. Left: CFD showing a high PD on the dome of the aneurysm. Right: Microsurgical findings showing a thin-walled region matching the high PD.

cases. They performed CFD analysis for 100 aneurysms, including 87 unruptured and 13 ruptured aneurysms of the internal carotid artery (ICA) and MCA. For the ruptured aneurysms, they were able to perform CFD analysis using the images that were taken before their rupture because the aneurysms ruptured during observation. Their results indicated that the PLc was statistically significantly lower in ruptured aneurysms, but statistical significance of a WSS difference was noted only for ICA cases. Takao et al. concluded that aneurysms likely to rupture have geometries in which blood flows easily and are hemodynamically stable. This hemodynamic stability may reduce natural vessel wall resistance to flow changes such as rapid blood pressure increase. As a result of exceeding the resistance limit, aneurysms may rupture. They hypothesized that a region with high PLc undergoes aneurysmal growth to change its shape in order to avoid interfering with and adapting to the blood flow. ${ }^{37}$ This is the "remodeling process" that eventually will reach a stable hemodynamic condition and thereafter will transition from a low to a high risk of rupture. In addition, Kono and Terada ${ }^{22}$ reported a PLc elevation from before to after carotid artery stenting, which was performed on unruptured anterior communicating artery aneurysms with pseudo-occlusions of the ICA. They also showed that the aneurysms stabilized after the PLc change, as they remained unchanged morphologically after stent deployment.

A thin aneurysmal wall is considered a risky condition for an unruptured aneurysm (Fig. 2). Kadasi et al. ${ }^{18}$ evaluated the surgical findings of aneurysmal wall condition and the role of WSS. They found that a thin wall was associated with low WSS. Suzuki et al. ${ }^{35}$ reported surgical findings of wall condition and CFD parameters in 50 MCA aneurysms. They found that the maximum PD corresponded to aneurysmal thin-walled regions in $82.0 \%$ of the $50 \mathrm{MCA}$ aneurysms, identified during craniotomy. A cutoff value was also introduced to estimate the existence of thin-walled regions by receiver operating characteristic analysis. This study compared real wall conditions observed through the surgical microscope and a CFD colorcoded image. The accuracy rate of predicting a thin-wall condition in this study was approximately $82 \%$. Additional prospective data analysis may be required to find valid parameters for clinical application. 

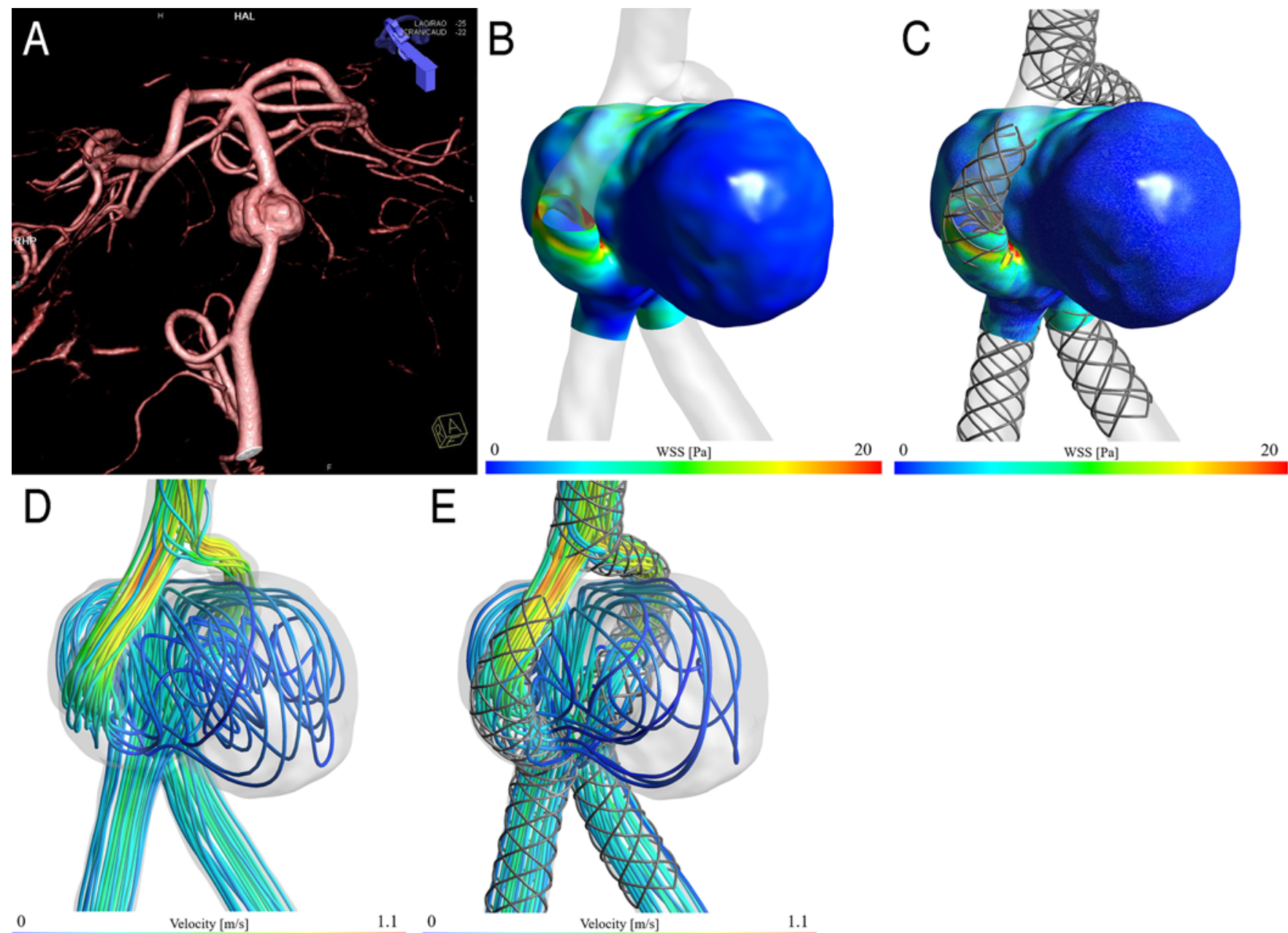

1.10

Velocity $[\mathrm{m} / \mathrm{s}]$ 1.1

FIG. 3. Images obtained in a 71-year-old woman who presented with a 13-mm basilar fenestration unruptured aneurysm. A: Three-dimensional angiogram before stent-assisted coil embolization. B-E: CFD simulation of double-barrel stent placement on the fenestrated bilateral basilar arteries: prestent WSS (B), poststent WSS (C), prestreamline (D), and poststreamline (E). The CFD simulation shows a slight reduction of WSS and flow (streamline) after stent placement.

\section{Role of CFD in Clinical Practice \\ Anticipation of Aneurysm Rupture}

Determining the fate of a patient with an aneurysm is the most difficult goal to achieve, and obviously the CFD technique is not the crystal ball. Aneurysm rupture is a multifactorial phenomenon that may not be predicted by a single parameter. The effects of mental and physical stress may contribute as well. Blood pressure elevation can be a trigger for rupture. But some other factors such as aneurysm size, location, presence of daughter sac, and certain other morphological conditions can be classified as high or low risk in terms of rupture using current CFD technology.

\section{Simulation of Endovascular Devices}

The most promising and feasible application of CFD may be endovascular device evaluation or development (Fig. 3, Videos 1 and 2).

VIDEO 1. Clip showing simulation (streamline) before FD placement on an aneurysm. Copyright Yuichi Murayama. Published with permission. Click here to view.
VIDEO 2. Clip showing simulation (streamline) after FD placement on an aneurysm, demonstrating significant reduction of flow in the aneurysm. Copyright Yuichi Murayama. Published with permission. Click here to view.

CFD analysis has also been performed to evaluate the effectiveness of devices such as flow diverter (FD) stents or embolic coils, by observing the blood flow changes between pre- and post-device application in treated aneurysms. ${ }^{8,10,26,33,34}$ Paliwal et al. ${ }^{26}$ analyzed the blood flow of FD stented aneurysms for 15 cases, including 10 complete occlusions and 5 partial occlusions. The results showed that a higher reduction rate in vortex core line length and energy loss occurred for the complete occlusion cases. Furthermore, Damiano et al. ${ }^{8}$ discussed the effectiveness of an FD stent-and-coil adjunctive technique from the results of CFD analysis. They indicated a possibility that adjunctive coils added no additional inflow reduction compared with a single FD until coil packing density exceeded a specific stasis in the aneurysms. Estimation of recanalization risk has also been attempted using CFD. Fujimura et al. ${ }^{11}$ performed CFD analysis for 100 coil-embolized aneurysms, 
including 26 repeat treatments due to recanalization and 74 aneurysms that were not re-treated. They developed a formula called "re-treatment predictor" to predict the risk of aneurysmal re-treatment using logistic regression. The predictor included hemodynamic parameters, morphological parameters, and clinical information, and PD was also introduced as one of the main risk factors of aneurysmal re-treatment. Sugiyama et al. ${ }^{32}$ reported that mass flow rate into the aneurysm before coiling was a significant risk factor for recanalization in basilar tip aneurysms; however, aneurysm size was statistically significantly larger for recanalized aneurysms.

\section{Limitations of CFD Studies}

In almost all CFD studies involving a large number of aneurysms, no biological information was utilized. Only morphological data, without input of actual blood pressure blood viscosity and the dynamic change in those biological factors, were considered. In particular, blood is assumed to be a Newtonian fluid in most simulations for cerebral arteries. Some researchers concluded that the difference between a Newtonian and a non-Newtonian blood model was not significant as the changes noted among varying aneurysm morphologies or WSS with these two models were similar on the aneurysm wall., ${ }^{938}$ However, the importance of their difference in CFD simulation has been studied. Hippelheuser et al..$^{15}$ investigated the effect of assuming a constant viscosity for cases with a bleb. They concluded that adopting a non-Newtonian viscosity model highlighted the hemodynamic differences induced by the presence of a bleb and improved the discriminant statistics in rupture prediction. Suzuki et al.$^{36}$ first compared the CFD simulation results of the Newtonian viscosity model with those of actual viscosity data obtained from blood sample data of healthy volunteers. They concluded that CFD using either the common Newtonian or non-Newtonian viscosity assumption could lead to values different from those of the patient-specific viscosity model for hemodynamic parameters such as WSS.

Although CFD simulations may be helpful in understanding and potentially designing endovascular devices (such as FDs), the wall characteristics and geometry of the parent artery may change after device deployment. Although these changes must have a great influence on numerous dynamic forces that involve the aneurysm and the parent artery interface, the aneurysm and parent artery were assumed to be rigid in most studies. In addition, it is highly possible that the rigid wall assumption may also affect the hemodynamics or dynamic forces on the aneurysm wall surface. Detailed research on these issues is limited because of the difficulties in simulating the distinct properties of vessel hysteresis, which most likely change from a normal vessel to an abnormal vessel.

While many hemodynamic parameters were investigated to estimate or evaluate aneurysm status, a wide variety of analytical tools and methods were introduced for CFD analysis, including segmentation tools, analysis software, and computational conditions. We also need to consider the effect that is produced from the use of different tools and methods. Berg et al. ${ }^{2}$ investigated the effect of segmentation approaches when generating the an- eurysmal geometry data. The participants were from 26 institutions around the globe that had the ability to generate stereolithography (STL) data from DICOM data, and they individually generated STL data of cerebral arteries, including aneurysms from the same DICOM data using their own methods. Despite the starting points being completely the same (i.e., the same DICOM data was inputted), the final STL data were markedly different. Because the results of CFD analysis rely heavily on geometry, each institution would have calculated different values of hemodynamic parameters, even if they were requested to perform identical tasks from that point. Many researchers have considered various approaches using CFD to evaluate aneurysms, however, currently a unified view has not yet been reached. The number of analyzed CFD cases is approximately 200 at maximum. The number of ruptured aneurysm analyses using images that were obtained before rupture is especially limited. Because each research institution has different methodology for CFD analysis, including segmentation and computational conditions, the analyzed hemodynamic values cannot simply be compared with each other. If we consider using CFD as a risk assessment tool for aneurysmal rupture in the future, it is necessary to perform a large-scale multicenter CFD study for unruptured and ruptured aneurysms that uses images before rupture. In addition, there is a need to standardize the CFD analysis method to a level to be able to guarantee the quality and comparability of hemodynamic results.

It is important to keep in mind that CFD data alone cannot be an absolute predictor of rupture. A patient's biological and genetic factors can also play very important roles in aneurysm rupture.

\section{Conclusions}

Although controversy remains regarding which risk factors contribute to predicting aneurysm rupture, CFD can provide additional parameters to assess the risk of rupture. This technology can potentially contribute to clinical decision-making or evaluation of endovascular devices for efficacy.

\section{Acknowledgments}

We thank Kostadin Karagiozov, MD, PhD, for manuscript preparation.

\section{References}

1. Aburakawa D, Fujimura M, Niizuma K, Sakata H, Endo H, Tominaga T: Navigation-guided clipping of a de novo aneurysm associated with superficial temporal artery-middle cerebral artery bypass combined with indirect pial synangiosis in a patient with moyamoya disease. Neurosurg Rev 40:517-521, 2017

2. Berg P, Voß S, Saalfeld S, Janiga G, Bergersen AW, ValenSendstad K, et al: Multiple Aneurysms AnaTomy CHallenge 2018 (MATCH): Phase I: Segmentation. Cardiovasc Eng Technol 9:565-581, 2018

3. Brinjikji W, Chung BJ, Jimenez C, Putman C, Kallmes DF, Cebral JR: Hemodynamic differences between unstable and stable unruptured aneurysms independent of size and location: a pilot study. J Neurointerv Surg 9:376-380, 2017

4. Castro MA, Putman CM, Sheridan MJ, Cebral JR: Hemody- 
namic patterns of anterior communicating artery aneurysms: a possible association with rupture. AJNR Am J Neuroradiol 30:297-302, 2009

5. Cebral JR, Mut F, Weir J, Putman C: Quantitative characterization of the hemodynamic environment in ruptured and unruptured brain aneurysms. AJNR Am J Neuroradiol 32:145-151, 2011

6. Cebral JR, Mut F, Weir J, Putman CM: Association of hemodynamic characteristics and cerebral aneurysm rupture. AJNR Am J Neuroradiol 32:264-270, 2011

7. Cebral JR, Vazquez M, Sforza DM, Houzeaux G, Tateshima S, Scrivano E, et al: Analysis of hemodynamics and wall mechanics at sites of cerebral aneurysm rupture. J Neurointerv Surg 7:530-536, 2015

8. Damiano RJ, Ma D, Xiang J, Siddiqui AH, Snyder KV, Meng $\mathrm{H}$ : Finite element modeling of endovascular coiling and flow diversion enables hemodynamic prediction of complex treatment strategies for intracranial aneurysm. J Biomech 48:3332-3340, 2015

9. Fisher C, Rossmann JS: Effect of non-Newtonian behavior on hemodynamics of cerebral aneurysms. J Biomech Eng 131:091004, 2009

10. Fujimura S, Takao H, Suzuki T, Dahmani C, Ishibashi T, Mamori $\mathrm{H}$, et al: Hemodynamics and coil distribution with changing coil stiffness and length in intracranial aneurysms. J Neurointerv Surg 10:797-801, 2018

11. Fujimura S, Takao H, Suzuki T, Dahmani C, Ishibashi T, Mamori H, et al: A new combined parameter predicts retreatment for coil-embolized aneurysms: a computational fluid dynamics multivariable analysis study. J Neurointerv Surg 10:791-796, 2018

12. Fukazawa K, Ishida F, Umeda Y, Miura Y, Shimosaka S, Matsushima S, et al: Using computational fluid dynamics analysis to characterize local hemodynamic features of middle cerebral artery aneurysm rupture points. World Neurosurg 83:80-86, 2015

13. Geers AJ, Morales HG, Larrabide I, Butakoff C, Bijlenga P, Frangi AF: Wall shear stress at the initiation site of cerebral aneurysms. Biomech Model Mechanobiol 16:97-115, 2017

14. Hassan T, Timofeev EV, Saito T, Shimizu H, Ezura M, Matsumoto Y, et al: A proposed parent vessel geometry-based categorization of saccular intracranial aneurysms: computational flow dynamics analysis of the risk factors for lesion rupture. J Neurosurg 103:662-680, 2005

15. Hippelheuser JE, Lauric A, Cohen AD, Malek AM: Realistic non-Newtonian viscosity modelling highlights hemodynamic differences between intracranial aneurysms with and without surface blebs. J Biomech 47:3695-3703, 2014

16. Hodis S, Uthamaraj S, Lanzino G, Kallmes DF, DragomirDaescu D: Computational fluid dynamics simulation of an anterior communicating artery ruptured during angiography. J Neurointerv Surg 6:e14, 2014

17. Jing L, Fan J, Wang Y, Li H, Wang S, Yang X, et al: Morphologic and hemodynamic analysis in the patients with multiple intracranial aneurysms: ruptured versus unruptured. PLoS One 10:e0132494, 2015

18. Kadasi LM, Dent WC, Malek AM: Colocalization of thinwalled dome regions with low hemodynamic wall shear stress in unruptured cerebral aneurysms. J Neurosurg 119:172-179, 2013

19. Kono K, Fujimoto T, Shintani A, Terada T: Hemodynamic characteristics at the rupture site of cerebral aneurysms: a case study. Neurosurgery 71:E1202-E1209, 2012

20. Kono K, Shintani A, Fujimoto T, Terada T: Stent-assisted coil embolization and computational fluid dynamics simulations of bilateral vertebral artery dissecting aneurysms presenting with subarachnoid hemorrhage: case report. Neurosurgery 71:E1192-E1201, 2012

21. Kono K, Terada T: Hemodynamics of 8 different configura- tions of stenting for bifurcation aneurysms. AJNR Am J Neuroradiol 34:1980-1986, 2013

22. Kono K, Terada T: Treatment strategy and follow-up evaluation for an unruptured anterior communicating artery aneurysm associated with pseudo-occlusion of the internal carotid artery using computational fluid dynamics simulations. Turk Neurosurg 24:111-116, 2014

23. Meng H, Tutino VM, Xiang J, Siddiqui A: High WSS or low WSS? Complex interactions of hemodynamics with intracranial aneurysm initiation, growth, and rupture: toward a unifying hypothesis. AJNR Am J Neuroradiol 35:1254-1262, 2014

24. Miura Y, Ishida F, Umeda Y, Tanemura H, Suzuki H, Matsushima S, et al: Low wall shear stress is independently associated with the rupture status of middle cerebral artery aneurysms. Stroke 44:519-521, 2013

25. Omodaka S, Sugiyama S, Inoue T, Funamoto K, Fujimura M, Shimizu H, et al: Local hemodynamics at the rupture point of cerebral aneurysms determined by computational fluid dynamics analysis. Cerebrovasc Dis 34:121-129, 2012

26. Paliwal N, Damiano RJ, Davies JM, Siddiqui AH, Meng H: Association between hemodynamic modifications and clinical outcome of intracranial aneurysms treated using flow diverters. Proc SPIE Int Soc Opt Eng 10135:10135F, 2017

27. Qian Y, Takao H, Umezu M, Murayama Y: Risk analysis of unruptured aneurysms using computational fluid dynamics technology: preliminary results. AJNR Am J Neuroradiol 32:1948-1955, 2011

28. Ramachandran M, Retarekar R, Raghavan ML, Berkowitz B, Dickerhoff B, Correa T, et al: Assessment of image-derived risk factors for natural course of unruptured cerebral aneurysms. J Neurosurg 124:288-295, 2016

29. Riccardello GJ Jr, Shastri DN, Changa AR, Thomas KG, Roman M, Prestigiacomo CJ, et al: Influence of relative residence time on side-wall aneurysm inception. Neurosurgery 83:574-581, 2018

30. Schneiders JJ, Marquering HA, van Ooij P, van den Berg R, Nederveen AJ, Verbaan D, et al: Additional value of intraaneurysmal hemodynamics in discriminating ruptured versus unruptured intracranial aneurysms. AJNR Am J Neuroradiol 36:1920-1926, 2015

31. Sforza DM, Kono K, Tateshima S, Viñuela F, Putman C, Cebral JR: Hemodynamics in growing and stable cerebral aneurysms. J Neurointerv Surg 8:407-412, 2016

32. Sugiyama S, Niizuma K, Sato K, Rashad S, Kohama M, Endo $\mathrm{H}$, et al: Blood flow into basilar tip aneurysms: a predictor for recanalization after coil embolization. Stroke 47:2541-2547, 2016

33. Suzuki T, Takao H, Fujimura S, Dahmani C, Ishibashi T, Mamori $\mathrm{H}$, et al: Relationships between geometrical parameters and mechanical properties for a helical braided flow diverter stent. Technol Health Care 25:611-623, 2017

34. Suzuki T, Takao H, Fujimura S, Dahmani C, Ishibashi T, Mamori H, et al: Selection of helical braided flow diverter stents based on hemodynamic performance and mechanical properties. J Neurointerv Surg 9:999-1005, 2017

35. Suzuki T, Takao H, Suzuki T, Kambayashi Y, Watanabe M, Sakamoto H, et al: Determining the presence of thin-walled regions at high-pressure areas in unruptured cerebral aneurysms by using computational fluid dynamics. Neurosurgery 79:589-595, 2016

36. Suzuki T, Takao H, Suzuki T, Suzuki T, Masuda S, Dahmani $\mathrm{C}$, et al: Variability of hemodynamic parameters using the common viscosity assumption in a computational fluid dynamics analysis of intracranial aneurysms. Technol Health Care 25:37-47, 2017

37. Takao H, Murayama Y, Otsuka S, Qian Y, Mohamed A, Masuda S, et al: Hemodynamic differences between unruptured and ruptured intracranial aneurysms during observation. Stroke 43:1436-1439, 2012 
38. Valencia AA, Guzmán AM, Finol EA, Amon CH: Blood flow dynamics in saccular aneurysm models of the basilar artery. J Biomech Eng 128:516-526, 2006

39. Xiang J, Natarajan SK, Tremmel M, Ma D, Mocco J, Hopkins LN, et al: Hemodynamic-morphologic discriminants for intracranial aneurysm rupture. Stroke 42:144-152, 2011

40. Xiang J, Yu J, Snyder KV, Levy EI, Siddiqui AH, Meng H: Hemodynamic-morphological discriminant models for intracranial aneurysm rupture remain stable with increasing sample size. J Neurointerv Surg 8:104-110, 2016

41. Zhang Y, Jing L, Liu J, Li C, Fan J, Wang S, et al: Clinical, morphological, and hemodynamic independent characteristic factors for rupture of posterior communicating artery aneurysms. J Neurointerv Surg 8:808-812, 2016

\section{Disclosures}

The authors report no conflict of interest concerning the materials or methods used in this study or the findings specified in this paper.

\section{Author Contributions}

Conception and design: Murayama. Acquisition of data: Suzuki, Takao. Analysis and interpretation of data: Fujimura. Drafting the article: Fujimura.

\section{Supplemental Information}

Videos

Video 1. https://vimeo.com/335122082.

Video 2. https://vimeo.com/335122189.

\section{Correspondence}

Yuichi Murayama: The Jikei University School of Medicine, Tokyo, Japan. ymurayama@jikei.ac.jp. 